\title{
Philosophiques
}

\section{L'intuition est-elle une attitude propositionnelle ?}

\section{GUILLAUME FRÉCHETTE}

Volume 44, numéro 1, printemps 2017

Nature, rôle et importance des intuitions

URI : https://id.erudit.org/iderudit/1040325ar

DOI : https://doi.org/10.7202/1040325ar

Aller au sommaire du numéro

\section{Éditeur(s)}

Société de philosophie du Québec

ISSN

0316-2923 (imprimé)

1492-1391 (numérique)

Découvrir la revue

Citer cet article

Fréchette, G. (2017). L'intuition est-elle une attitude propositionnelle? Philosophiques, 44(1), 11-30. https://doi.org/10.7202/1040325ar

\section{Résumé de l'article}

Il est généralement admis dans la littérature analytique sur l'intuition que celle-ci est principalement, ou même fondamentalement, une attitude propositionnelle. Partant de là, elle est aussi souvent caractérisée comme une croyance que $P$, comme la formation d'une croyance sans inférence que $P$, comme une impression que $P$ (seeming : Bealer 1998), comme une impression intellectuelle que $P$ (Huemer 2001 ; 2005), comme l'attitude consistant à être poussé, mu (pushed by) par $P$ (Koksvik 2011). Dans tous les cas, la spécificité de l'intuition reposerait au moins en partie sur les propriétés doxastiques qui la distingue d'autres attitudes propositionnelles, comme savoir que $P$ ou douter que $P$.

Cette caractérisation de l'intuition semble à première vue incommensurable avec le concept d'intuition discuté dans la tradition phénoménologique, où l'intuition est caractérisée comme ce type d'expérience qui rend les objets présents (ou même parfois qui offre un accès privilégié à la vérité que $P$ ), et peut certes être caractérisée en termes d'attitude propositionnelle, mais ne l'est pas essentiellement.

Dans ce qui suit, je soulève quelques problèmes auxquels fait face la conception de l'intuition comme attitude propositionnelle. Partant de là, j'aimerais suggérer qu'en amendant cette idée, on peut développer une théorie de l'intuition qui peut employer de manière fructueuse les ressources de la phénoménologie et de la philosophie analytique. Cette suggestion montre que l'incommensurabilité des conceptions analytiques et phénoménologiques de l'intuition est superficielle, plus superficielle que ne le laissent entendre ses défenseurs respectifs.
Ce document est protégé par la loi sur le droit d'auteur. L’utilisation des services d'Érudit (y compris la reproduction) est assujettie à sa politique d'utilisation que vous pouvez consulter en ligne.

https://apropos.erudit.org/fr/usagers/politique-dutilisation/ 


\title{
L'intuition est-elle une attitude propositionnelle?
}

\author{
GUILLAUME FRÉCHETTE
}

Université de Salzbourg

RÉSUMÉ. - Il est généralement admis dans la littérature analytique sur l'intuition que celle-ci est principalement, ou même fondamentalement, une attitude propositionnelle. Partant de là, elle est aussi souvent caractérisée comme une croyance que $P$, comme la formation d'une croyance sans inférence que $P$, comme une impression que $P$ (seeming: Bealer 1998), comme une impression intellectuelle que $P$ (Huemer 2001; 2005), comme l'attitude consistant à être poussé, mu (pushed by) par $P$ (Koksvik 2011). Dans tous les cas, la spécificité de l'intuition reposerait au moins en partie sur les propriétés doxastiques qui la distingue d'autres attitudes propositionnelles, comme savoir que $P$ ou douter que $P$.

Cette caractérisation de l'intuition semble à première vue incommensurable avec le concept d'intuition discuté dans la tradition phénoménologique, où l'intuition est caractérisée comme ce type d'expérience qui rend les objets présents (ou même parfois qui offre un accès privilégié à la vérité que $P$ ), et peut certes être caractérisée en termes d'attitude propositionnelle, mais ne l'est pas essentiellement.

Dans ce qui suit, je soulève quelques problèmes auxquels fait face la conception de l'intuition comme attitude propositionnelle. Partant de là, j'aimerais suggérer qu'en amendant cette idée, on peut développer une théorie de l'intuition qui peut employer de manière fructueuse les ressources de la phénoménologie et de la philosophie analytique. Cette suggestion montre que l'incommensurabilité des conceptions analytiques et phénoménologiques de l'intuition est superficielle, plus superficielle que ne le laissent entendre ses défenseurs respectifs.

ABSTRACT. - It is generally acknowledged in the analytic literature on intuitions that these are generally, or even fundamentally, propositional attitudes. For this reason, intuitions are often characterized as beliefs that $P$, as seeming that $P$ (Bealer 1998), as the intellectual seeming that $P$ (Huemer $2001 ; 2005$ ), or as the attitude of being pushed by $P$ (Koksvik 2011). In all cases, the specificity of intuitions would consist at least in part in the doxastic properties that distinguishes them from other propositional attitudes, such as knowing that $P$ or doubting that $P$.

At first glance, this characterization of intuitions seems incommensurable with the concept of intuition discussed in the phenomenological tradition, where intuition is characterized as the type of experience that make objects present to us (or even sometimes as the type of experience which offers a priviledged access to the truth that $P$ ). While intuitions in this sense may be characterized as propositional attitudes, it doesn't imply that they fundamentally are propositional.

In the following paper, I raise some problems which faces the conception of intuitions as propositional attitudes. I would suggest that amending this idea allows to develop a theory of intuition which can use fruitfully both the resources of phenomenology and analytic philosophy. As a consequence, the alleged

PHILOSOPHIQUES 44/1 — Printemps 2017, p. 11-30 
incommensurability of analytical and phenomenological conceptions of intuitions appears to be more superficial than it is usually taken to be defenders of intuitions as propositional attitudes.

\section{L'intuition. Une attitude propositionnelle?}

Lorsqu'on parle de l'intuition en philosophie analytique contemporaine, on veut souvent parler en fait d'une attitude propositionnelle. À côté d'attitudes propositionnelles comme penser que $P$, souhaiter que $P$, on retrouverait donc un type d'attitude propositionnelle comme celle d'avoir l'intuition $q u e ~ P$, où cette attitude est fondamentalement propositionnelle. Appelons cette thèse la thèse $A$.

Il y a cependant un sens dans lequel on parle d'intuition qui semble récurrent dans l'histoire de la philosophie. Suivant Descartes, Locke avançait par exemple que les intuitions donnent accès à des connaissances sans aucune médiation d'autres idées. Hume voyait dans l'intuition une forme de connaissance immédiate des axiomes logiques et mathématiques, et, avec Locke et Berkeley, il défendait également l'idée que les objets individuels nous sont donnés dans l'intuition. Suivant cette compréhension de l'intuition, la connaissance intuitive est celle qui est fondée dans l'intuition des objets individuels. C'est cette idée des empiristes qui est reprise par Brentano lorsque celui-ci décrit les représentations intuitives - celles de qualités comme la couleur et le son, par exemple - comme étant à la base de nos représentations conceptuelles. Et bien qu'il n'entérine pas la conception empiriste de Brentano, Husserl reprend aussi cette idée lorsqu'il parle d'intuition catégoriale, une forme de connaissance des essences qui ne procède pas par l'abstraction à partir des objets concrets qui nous sont donnés dans l'intuition sensible. Or dans tous les cas mentionnés, de Descartes à Husserl, personne ne défend visiblement la thèse $A$. En fait, tous semblent d'accord avec le fait que l'intuition est l'attitude mentale qui précède l'abstraction, et dans laquelle des contenus sont donnés de manière immédiate: ces contenus peuvent être représentationnels ou propositionnels, mais dans un cas comme dans l'autre, la propositionnalité n'est pas constitutive de ce qu'est l'intuition.

Certains défenseurs de la thèse $\mathrm{A}$ ajouteront que si j'ai l'intuition que $P$, c'est que je crois que $P$. Donc l'intuition est un type d'attitude propositionnelle qui en présuppose un autre: la croyance. Parmi les défenseurs de cette version de la thèse $\mathrm{A}$, on retrouve notamment David Lewis (I983, $\mathrm{x}$ ). Nous appellerons ici cette position position 1 (posi).

D'autres, comme Ernest Sosa (I998), pensent plutôt que c'est une disposition à croire. Si j'ai l'intuition que $P$, c'est que je suis disposé à croire que $P$. C'est une option dont je ne discuterai pas ici, mais qui est simplement mentionnée comme autre solution possible. Appelons-la position 2 (pos2).

Une troisième voie, popularisée depuis les dernières années, consiste à dire qu'avoir l'intuition que $P$ n'est pas croire que $P$ : c'est plutôt une atti- 
tude propositionnelle sui generis, qui fonde la croyance que $P$. Du coup, contrairement à (posi), elle ne la présuppose pas. Nous appellerons cette troisième option de défense de la thèse $\mathrm{A}$ la position 3 (pos3). C'est la position que beaucoup défendent depuis les quinze dernières années, en commençant par Bealer (1998), Huemer (200I; 2005), et plus récemment Chudnoff (20I3), qui disent que l'intuition est un seeming, une "apparence", ou une «impression» qu'une proposition est vraie, etc. Plusieurs des contributions réunies dans Tucker (20I3) sur le conservatisme phénoménal et le concept de «seeming» vont d'ailleurs dans cette direction. Le conservatisme phénoménal affirme que, s'il semble à $S$ que $P$, alors en l'absence d'une évidence contraire (defeater), $S$ est donc justifié de croire que $P^{1}$. Les «évidences contraires» sont de deux sortes. La première est illustrée par des cas d'illusions comme celle de Müller-Lyer, où les droites nous semblent être de longueurs inégales alors qu'on voit tout de suite qu'elles sont de même longueur lorsque les pointes obtuses et aiguës sont retirées. L'évidence contraire - à savoir que les lignes sont, contre toute apparence, de longueur égale — vient alors réfuter notre impression. Le second type d'évidence contraire vient plutôt rectifier notre impression. C'est le cas par exemple lorsque, naïvement, nous voyons un objet comme étant rouge, une impression qui doit être rectifiée face à l'évidence contraire apportée par exemple par la connaissance qu'un éclairage d'un type de luminosité rougeâtre fait apparaître cet objet de cette couleur. Dans ce deuxième cas, l'évidence contraire a une portée uniquement rectificatrice, car elle ne nous engage pas à croire que l'objet en question n'est pas réellement rouge sous des conditions d'éclairage normales. Bref, pour les défenseurs du conservatisme phénoménal, la connaissance est une croyance vraie justifiée par la façon dont les choses nous semblent être (en l'absence d'évidence contraire).

Dans les trois positions introduites ici, on parle de l'intuition en termes d'attitude propositionnelle pour une bonne raison: on veut dire que l'intuition est une source de l'évidence que $P$ est vrai. J'appellerai cela la vertu épistémique de l'intuition. Si l'intuition a une vertu épistémique, il semble que la réponse à la question posée dans le titre de cet article soit trivialement «oui».

J'ai deux doutes sur cette réponse triviale: d'abord on peut se demander si la vertu épistémique des intuitions nécessite qu'on comprenne celles-ci comme des attitudes propositionnelles: si on peut montrer qu'il y a des formes de connaissance qui ne sont pas propositionnelles, on peut montrer que l'intuition n'est pas nécessairement une attitude propositionnelle. Après tout, on parle bien de connaissance par accointance (Russell), ou de prise de connaissance (Kenntnisnahme) chez certains philosophes austro-allemands, lesquelles sont des formes de connaissance qui ont très clairement des vertus épistémiques mais ne sont pas, du moins à première vue, propositionnelles.

1. Comparer Huemer $(2007,30)$. 
Si ces formes de connaissance sont de nature intuitive, alors on peut penser que les défenseurs de la thèse $\mathrm{A}$ échouent à rendre compte de manière appropriée du phénomène décrit par les empiristes et les phénoménologues. Bref, on pourrait sauver la vertu épistémique des intuitions sans accepter la thèse $\mathrm{A}$.

Ensuite, même en acceptant la position plus modérée que l'intuition n'est pas une sorte de croyance, donc en défendant (pos3), on ne sait pas très bien en quoi un seeming est fondamentalement une attitude propositionnelle; les tenants de (pos3) parlent de seeming (Bealer), d'intellectually seeming (Huemer), ou de l'attitude qui consiste à «être poussé vers $P$ » (Koksvik, 20I I), d'avoir la représentation de $P$ comme vrai (Chudnoff). Or toutes les métaphores employées pour décrire cette attitude sui generis ne semblent pas avoir quelque chose de strictement propositionnel. Lorsque j'observe ce qui me semble être une maison de loin sur la route, puis que je m'aperçois en m'approchant que ce n'était qu'un panneau publicitaire, on peut effectivement dire que dans un premier temps, j'étais "poussé vers» ou "penchais » vers la proposition «cet objet au loin est une maison». Mais on peut aussi bien dire, il me semble, que l'inclination qui se manifeste à ce moment découle de l'appréhension que j'ai de l'objet à ce moment: que je penche vers la proposition "cet objet au loin est une maison" semble en ce sens conditionné par la façon dont j'appréhende l'objet. Cette appréhension dépend directement et largement des modes d'apparition de l'objet en question: des facteurs aussi divers et multiples que des conditions atmosphériques, des troubles de vision, les formes et couleurs particulières de l'objet, certaines associations automatiques réalisées en fonction d'un contexte qui s'apparente à un autre, etc., influencent l'appréhension des contenus sensoriels qui me sont donnés. Ils orientent cette dernière dans une direction plutôt qu'une autre. Pour rester dans la métaphore des défenseurs de (pos3), on pourrait très bien dire en un sens analogue que ces facteurs qui sont déterminants pour le mode d'apparition de l'objet ou de l'état de choses me "poussent à voir» une maison dans un pré. Bref, prima facie, la "semblance », et même le fait de "pencher pour » ou être "incliné à » ne semblent pas exprimer d'emblée une attitude propositionnelle sui generis. On peut très bien vouloir expliquer le phénomène mental en jeu qui est exprimé par l'énoncé «il me semble que c'est une maison " prononcé dans le contexte en question en termes d'attitude propositionnelle - et être justifié de le faire — mais on peut tout aussi bien être justifié d'expliquer le même phénomène par l'appréhension particulière de l'objet. C'est cette deuxième explication qui m'intéresse ici.

La plupart des défenseurs de (pos3) ne partagent pas cette intuition sur la nature des intuitions. Tucker (2013), qui pourtant est un défenseur de la force justificative des seemings, insiste justement pour en faire des attitudes propositionnelles. "There are [...] many accounts of perceptual experience which hold that perceptual experiences lack propositional 
content [...] I think those accounts are more naturally construed as accounts about sensations rather than seemings" (Tucker 20I3, 5). On retrouve ce genre de refus expéditif, sans la moindre tentative de l'appuyer de manière argumentative, également chez Pust (20I2) et Koksvik (20I I). J'appellerai cette intuition sur la nature des intuitions le "primitivisme propositionnel » de (pos3).

Dans ce qui suit, je vais d'abord introduire quelques distinctions concernant différents types de connaissance en partant des positions des phénoménologues austro-allemands, afin d'isoler au moins un cas dans lequel on peut parler d'intuition non propositionnelle $(\mathbb{S} 2)$. Je vais ensuite expliquer en quoi cette conception, qu'on retrouve à la base notamment chez Brentano et Husserl, peut rendre compte des cas d'intuition d'objet et d'états de choses $(\mathbb{S} 3$ et 4$)$. En partant de Husserl et suivant une suggestion de Mulligan, je proposerai ensuite un moyen de rendre compte de la vertu épistémique des intuitions, tant celles qui portent sur les objets et les états de choses, en suggérant que les intuitions propositionnelles gagneraient à être expliquées en termes d'intuitions sur des états de choses $(\mathbb{5}$ ).

\section{Une double distinction}

Lorsqu'on parle de connaissance, on entend souvent par là une certaine définition classique de la connaissance comme croyance vraie et justifiée. $S$ sait que $P$ ssi $S$ est justifié de croire que $P$ et $P$ est vrai. Mais il y a d'autres formes de connaissances, et il y a d'autres conceptions de la connaissance. Russell parlait en I9Io de connaissance par accointance: j'ai une connaissance par accointance d'un objet lorsque je suis directement conscient de cet objet. $S$ a une accointance de $O$ est la relation converse de $O$ est présenté à $S$. Or Russell ne dit pas grand-chose de l'accointance, sinon que ce n'est pas une connaissance des vérités (bien qu'il puisse effectivement être le cas que toute connaissance par accointance soit accompagnée d'une connaissance par description). Et la plupart des philosophes analytiques qui se basent aujourd'hui sur le concept de Russell le prennent aussi pour un concept analytique, certains notamment pour défendre l'argument de la connaissance de Jackson.

Parmi ces autres conceptions de la connaissance, il y en a d'autres qui n'impliquent pas que la connaissance soit une croyance vraie justifiée, mais qu'elle soit plus fondamentale que la croyance. Au moins depuis « Knowledge first » de Williamson (2000), on peut penser que cette vieille idée a regagné en dignité. Les philosophes austro-allemands emploient par exemple «Erkennen » pour décrire un type de connaissance propre à l'acte de connaître (Erkennen), qu'on traduit aussi souvent par "acte d'appréhender ". Même si appréhender au sens d'Erkennen, c'est appréhender que $P$, appréhender que $P$ n'est pas croire que $P$.

On peut donc dire d'emblée que les Austro-Allemands sont au moins aussi des défenseurs de (pos3) en ce que l'appréhender est présupposé par le 
jugement (et la croyance) et en est distinct (Hildebrand I950, 8$)^{2}$. Mais beaucoup d'Austro-Allemands défendent plus que simplement (pos3), ils défendent le concept d'appréhension présenté dans la section I : pour eux, les objets de l'appréhension sont de toutes sortes: ce ne sont pas seulement les corrélats objectifs des propositions (les états de choses), mais ce sont également les corrélats objectifs de représentations (les objets), alors que les objets du jugement et de la croyance sont seulement des états de choses.

Une autre différence importante entre l'appréhension d'une part, et le jugement et la croyance d'autre part, est que l'appréhension n'est pas médiatisée par la signification, alors que le jugement et la croyance le sont. Comme le dit Reinach, dans l'appréhension un état de choses m'est présenté, et c'est sur cette base que se développe la croyance en l'état de choses; la croyance est fondée dans l'appréhension (Reinach I989, I20).

Une troisième différence qui découle de la différence entre l'appréhension et le jugement est que l' "Erkennen» est un acte, c'est donc un état mental épisodique; alors que savoir que $P$ ou croire que $P$ ne sont pas épisodiques, ce sont plutôt des états mentaux. Or cette forme fondamentale, épisodique, de la connaissance, ne se limite cependant pas à l'Erkennen. Certains phénoménologues comme Reinach, Hildebrand et Bassenge font valoir une autre forme de connaissance épisodique, qui est celle de la prise de connaissance (Kenntnisnahme) pour Hildebrand, ce que Bassenge appelle la hexis, l'attitude au sens plus général d'une tenue de nature cognitive (Haltung) ou l' "avoir connaissance" (Kenntnishaben). Son caractère épisodique n'est pas complètement assuré chez les phénoménologues. Pour Bassenge, elle se distingue de l'appréhension principalement par son caractère non épisodique: là où l'appréhension relève d'une "saisie ponctuelle» (Einschnappen), la prise de connaissance, ou l' "avoir connaissance» est un état mental doté d'une certaine persistance. ${ }^{3}$ Pour Hildebrand et Reinach, elle semble davantage à ranger du côté de la connaissance épisodique.

Indépendamment de son caractère épisodique ou non, l'«avoir connaissance» est un mode de connaissance plus riche que l'appréhension. Je sais par exemple que le génocide juif commis par les nazis est un des crimes les plus meurtriers du $\mathrm{Xx}^{\mathrm{e}}$ siècle et je peux décider, sur la base de cette connaissance, de m'opposer au nazisme, sans toutefois que l'état de choses exprimé dans la proposition "le génocide juif commis par les nazis est un des crimes les plus meurtriers du $\mathrm{Xx}^{\mathrm{e}}$ siècle » me soit donné sur la base d'une prise de connaissance. Dans la prise de connaissance, l'état de choses est donné en tant que fondé dans certains contenus précis, par exemple dans les innombrables rapports des survivants, les documents historiques, les installations avec lesquelles ces crimes ont été perpétrés, les conditions inhumaines

2. Sur les conceptions austro-allemandes de l'appréhension et de la connaissance, cf. Mulligan (20I4).

3. Voir Bassenge (1930). 
dans lesquelles les Juifs ont d'abord été maintenus, etc. ${ }^{4}$ Indépendamment du fait qu'elle fonde ou non une appréhension actuelle, il semble que la prise de connaissance donne un accès foncièrement différent - mieux informé, notamment - à son objet. Or si on accepte ce que les défenseurs de (pos3) disent être le propre de l'intuition, à savoir le fait d'être mu par la vérité d'une proposition, il n'y aurait aucune raison de faire la distinction entre une appréhension et une prise de connaissance. Pourtant, il semble que la prise de connaissance soit la seule des deux capable d'expliquer de manière substantielle ce que cela veut dire que d'être mu par la vérité de $P$ : la prise de connaissance donnant l'état de choses dans ses relations avec les contenus fondateurs, elle offre des outils plus précis pour expliquer comment on peut être mu par la vérité d'une proposition, ou comment une proposition peut nous sembler vraie, que ceux dont disposent les défenseurs de (pos3). Cette précision est redevable notamment du fait que les contenus fondateurs de l'état de choses dont on prend connaissance sont co-présents, des contenus qui sont souvent des contenus de représentations caractérisés par des degrés plus ou moins élevés du type d'intuitivité dont nous avons parlé dans la section I, en lien avec les empiristes et les phénoménologues.

Les phénoménologues que nous venons de mentionner considèrent qu'à chaque type d'objet correspond par essence un type de Kenntnisnahme déterminé (Hildebrand I9 I6, I 4 I, Husserl, 6 ${ }^{\mathrm{e}}$ RL sur l'intuition). La Kenntnisnahme est précisément ce type de connaissance plus primitif que la croyance dans lequel l'objet ou l'état de choses est donné d'une certaine manière: elle n'implique pas l'évidence de la connaissance de l'objet (Hildebrand I9I6, I44).

À ma connaissance, Brentano n'emploie pas le terme de Kenntnisnahme et ne développe pas non plus l'idée (et la rejette généralement dans sa psychologie descriptive) que certains des éléments de l'esprit soient des états et d'autres des actes. Son ontologie des éléments du mental est essentiellement constituée d'actes. Or Brentano affirme parfois que certaines représentations dites intuitives sont des prises de position, évidentes ou non, en faveur de l'objet représenté, des prises de position ou «acceptations» qui ne sont pas à proprement parler des jugements, bien qu'ils partagent avec les jugements la qualité d'acceptation. Ce type d'actes de connaissance est parfois décrit comme une "croyance instinctive et immédiate", distincte des activités cognitives supérieures. Comme le dit Marty, c'est un «acte qui contient deux parties mutuellement inséparables, l'intuition du phénomène physique et son acceptation assertorique ${ }^{5} »$. L'idée poursuivie par Brentano et Marty est que certains actes semblent être des acceptations ou prises de position instinctives, mues par une nécessité interne. Le cas pris en exemple est celui des jeunes enfants qui acceptent instinctivement comme tel ce qu'on

4. Comparer Hildebrand (I9I6), p. I49.

5. Marty, tel que cité par Kraus (I976). 
leur présente, souvent sans avoir même les capacités cognitives de discerner les cas où ils sont trompés des cas véridiques. Des cas comme celui où un nouveau-né tète l'auriculaire de la main de sa mère sans différencier le sein du doigt semblent être le genre de phénomène que Brentano et Marty ont en vue. Or on retrouverait dans des cas comme celui-ci la même situation que celle qui a lieu dans la représentation intuitive, à savoir une acceptation inconditionnelle et sans autre option de ce qui est représenté.

Enfin, lorsqu'il parle d'assomptions (Annahmen) comme pouvant saisir (Erfassen) un état de choses, Meinong développe lui aussi une conception de la connaissance non épisodique qui a une certaine parenté avec celle de la Kenntnisnabme des phénoménologues. On en arrive ainsi à une double distinction des formes de connaissance:

\begin{tabular}{|l|l|l|}
\hline & $\begin{array}{l}\text { Connaissance épisodique } \\
\text { (actes) }\end{array}$ & $\begin{array}{l}\text { Connaissance non } \\
\text { épisodique (états) }\end{array}$ \\
\hline Propositionnel & $\begin{array}{l}\text { Appréhender l'état de } \\
\text { choses exprimé par } P, \\
\text { juger que } P\end{array}$ & $\begin{array}{l}\text { Savoir que } P \text {, croire que } P, \\
\text { Annahme (assomption) } \\
\text { que } P .\end{array}$ \\
\hline Non propositionnel & $\begin{array}{l}\text { Appréhender A, avoir } \\
\text { l'intuition (aveugle ou non) } \\
\text { de A, Kenntnisnehmen de } \\
\text { A ou de l'état de choses } \\
\text { exprimé par } P\end{array}$ & Être en accointance avec A \\
\hline
\end{tabular}

\section{L'intuition chez Brentano et Husserl}

Si on accepte donc qu'il y a au moins quelque raison de penser, comme le suggèrent les Austro-Allemands, que la connaissance est peut-être quelque chose de plus primitif qu'une croyance vraie justifiée, qu'elle peut fonder de telles croyances, alors il n'est pas exclu que, même si elle doit absolument avoir une vertu épistémique, l'intuition appartienne à ce type de connaissance plus primitif que la croyance (contra posi). Et si cela est le cas, alors l'idée de voir l'intuition comme une attitude propositionnelle sui generis perd de l'intérêt si on peut montrer que les cas de connaissance plus primitive que la croyance (notamment la Kenntnisnabme) englobent, en plus des cas couverts par ce que certains appellent l'attitude propositionnelle sui generis, les cas qui fondent cette attitude. Bref, si on peut apporter quelque chose de plus au fameux «seeming " propositionnel, on pourrait même améliorer (pos3). Nous avons déjà dit à cet effet que la prise de connaissance avait l'avantage explicatif, face au seeming ou à l'inclination envers une proposition, de fournir des outils plus précis pour décrire ce qu'est une intuition, puisqu'elle nous donne l'état de choses dans ses relations avec les contenus fondateurs. Nous avons aussi suggéré que ces contenus avaient 
certaines propriétés dites d'intuitivité (au sens empiriste et phénoménologique du terme) qui pouvaient contribuer à déterminer plus précisément ce qui est en jeu dans les intuitions telles que décrites par les défenseurs de (pos3). Afin d'apprécier la nature de cette contribution, penchons-nous maintenant sur ces propriétés d'intuitivité.

Le concept d'intuition au sens d'intuitivité est au centre de la philosophie des Brentaniens; pour Brentano et Husserl, l'intuition est la forme la plus basique de l'expérience; pour Brentano, c'est le cas paradigmatique de la représentation, pour Husserl, c'est la procédure à la base de toute la phénoménologie $\left(6^{\mathrm{e}} \mathrm{RL}\right)$ : la phénoménologie fonctionne sur la base d'un "procédé intuitif» (intuitives Verfahren: Hua XIX/I, p. 6). De plus, l'intuition est immédiate, elle donne l'objet in propria persona ${ }^{6}$. Lorsque je vois la tour Eiffel «en propre», quelque chose m'est donné d'une manière directe et immédiate. Brentano caractérise justement cette immédiateté en termes de "représentations propres». Selon lui, les intuitions sont des représentations propres de contenu sensoriel. Une représentation propre est une représentation dont les parties du contenu sont des parties réellement attributives.

Une autre caractéristique commune à la conception des intuitions défendue par Brentano et Husserl est la parenté de l'intuition avec la perception. Les phénoménologues comme Brentano et Husserl caractérisent généralement l'intuition (Anschaunng) comme une forme particulière de perception, en opposition par exemple aux croyances intuitives, ou aux inclinations à croire, ou à tout autre attitude propositionnelle ressemblant aux croyances. En ce sens, pour Husserl et Brentano, les actes intuitifs sont perceptuels, bien que ce qui est considéré comme «perceptuel» (pour Husserl) dépasse ce qui tombe strictement dans le domaine de la perception sensorielle.

Ensuite, les intuitions ne sont pas infaillibles. Brentano et Husserl s'accordent sur le fait que l'intuitivité d'un acte n'est pas une garantie de l'infaillibilité de la perception. Si je vois ce que je pense être une femme dans un musée de cire, mon intuition sensorielle est grosso modo la même que celle que j'aurais eue si j'avais vu une véritable personne, peu importe que je voie dans les faits une poupée de cire ou une personne. De manière similaire, Brentano affirme qu'on peut avoir une intuition d'un carré rouge lorsqu'on est confronté à une figure géométrique colorée différemment .

6. Husserl, $5^{\mathrm{e}} \mathrm{RL}: \mathbb{S} 2, \mathbb{I}$ I $\mathbb{S}$ I4. Voir Hua XIX/I, p. $356 \mathrm{ff} ; 385 \mathrm{ff} ; 394 \mathrm{ff}$.

7. Selon Brentano, les intuitions sont normalement accompagnées par une interprétation (Deutung) de celles-ci. Les cas d'illusions d'optique l'illustrent le mieux: dans le cas de l'illusion de Müller-Lyer, les intuitions à la base de ma conviction que les lignes sont inégales ne sont pas en soi erronées - les lignes m'apparaissent inégales non pas parce que je les observerais incorrectement, mais parce que le contenu de la perception est interprété incorrectement. Sur ce cas, voir Brentano (I 892 ; I 893 ; I 894). Dans des cas analogues où les illusions d'optique ne sont pas concernées, voir Brentano (1959, 83sq.). 
Une dernière caractéristique commune à la conception des intuitions défendue par Brentano et Husserl est que les intuitions remplissent nos intentions de signification (erfüllen chez Husserl), ou satisfont nos intérêts (Interesse chez Brentano). Pour Husserl, l'intuition joue un rôle central dans la connaissance en vertu de sa capacité à remplir ce qui nécessite un remplissement. Selon lui, la perception est une interprétation de contenus intuitifs. Ces contenus remplissent, à un degré plus ou moins élevé, les intentions de signification. Dans les termes de Husserl, mon acte énonciatif exprimé par "les corbeaux sont noirs» a une intention de signification. L'énoncé implique des concepts - le concept d'un corbeau, le concept de quelque chose de noir - qui constituent la signification de l'intention et qui seraient les mêmes dans votre énoncé «les corbeaux sont noirs". L'idée que l'intuition a une fonction «remplissante» vient d'une idée développée dans les leçons de Brentano à Vienne, leçons aussi suivies par Husserl, et selon laquelle l'intérêt, une opération mentale distincte de la simple représentation, est satisfait ou rempli par les intuitions et leurs parties. En ce qu'elles peuvent être remplies par les intuitions correspondantes, les intentions husserliennes sont comme les phénomènes d'intérêt de Brentano, où l'intérêt est toujours dirigé vers une partie spécifique du contenu intuitif ${ }^{8}$.

Comme nous venons de le mentionner, l'intuition n'est pas seulement sensorielle pour Husserl. Elle vient en plusieurs variétés: en plus des intuitions sensorielles comme celles en jeu dans l'écoute d'un son ou dans la vision d'une couleur, il y a aussi des intuitions du général: en utilisant la méthode des variations eidétiques, je peux ainsi en arriver à une intuition du triangle en général. Dans ce cas, l'intuition sensorielle d'une couleur est accompagnée par l'intuition eidétique du triangle. L'intuition eidétique est un "voir» au sens général, "au sens universel [en tant que] conscience originalement présentative» (Idées directrices, $\mathbb{S}$ I9; Hua III/I, p. 4Isq).

L'intuition est aussi une expérience (ou conscience) immédiate. Husserl emploie le terme «intuition» ou sa forme adjectivale «intuitif» pour caractériser l'immédiateté d'une expérience. Comment doit-on comprendre cette immédiateté? Une façon de le faire serait de concevoir notre expérience comme elle nous est donnée et précédant toute interprétation qu'on lui fait

8. Sur la discussion, chez le jeune Husserl, du remplissement en termes d'intérêt, d'attention, ou de plaisir à remarquer - une terminologie empruntée à Brentano et à Stumpf, voir le volume XXII des Husserliana, et en particulier les Psychologische Studien de I 893, où il décrit la relation entre la représentation et l'intuition comme une relation entre la visée et le remplissement (Meinung und Erffüllung, Hua XXII, p. 292). Le remplissement d'une intuition est caractérisé comme une libération de la tension (Lösung der Spannung) (Hua XXII, 407, 4I I), comme une libération (Befreiung), comme une libération de l'inhibition (Erlösung der Hemmung: Hua XXII, 296), comme un allégement (Hua XXII, 4I 5). Mais il décrit aussi le remplissement comme un "plaisir à remarquer qui émane de l'objet» (Hua XXII, 293; 4II). Sur la description par Brentano de l'intérêt et de sa satisfaction, voir les cours de logique de Vienne (EL72), p. I23 Ioff. 
normalement subir. Prenons par exemple mon expérience particulière de boire du café: le café - disons un mélange corsé d'une sorte de grains particulière - est «immédiatement vécu» de la même manière primitive et immédiate que mon idée qu'il pourrait pleuvoir demain. L'adjectif «immédiat » dans ce contexte sert à souligner le fait que l'expérience n'a pas été abordée de manière réflexive. L'expérience immédiate est une expérience "sur le coup» de ce qui arrive au moment où cela arrive. L'immédiateté est une caractéristique qu'on retrouve dans toute intuition, et elle n'est pas dérivée de l'expérience sensorielle. Je fais «immédiatement » l'expérience du café non pas en vertu de l'amertume ressentie lorsque j'y goûte - bien qu'on fasse aussi l'expérience de l'amertume de manière immédiate - mais simplement parce que le café est l'objet de mon expérience. On a une expérience immédiate de tous les objets d'expérience — qu'ils soient sensibles ou non?

La fonction remplissante de l'intuition est souvent décrite par Husserl dans les Idées directrices par son caractère d'intuition originairement donatrice (originär gebende Intuition). La caractéristique «donatrice» s'applique au remplissement ou à la fonction remplissante des actes intuitions. Pour revenir à l'expérience du café, mon acte de penser le contenu de la proposition exprimée par «le café est un mélange éthiopien» est ce que Husserl appelle un acte signitif. Comme tel, pris isolément, c'est une intention vide. Dans mon expérience de goûter au café et d'y penser, on peut distinguer, suggère Husserl, entre deux moments: mon acte de penser signitif exprimé par «le café est un mélange éthiopien », et l'intuition remplissante, c'est-àdire mon expérience des propriétés qualitatives des mélanges éthiopiens, par exemple leur acidité et leur amertume qui les distingue par exemple d'un mélange brésilien. Une intention peut être plus ou moins optimalement remplie - il peut par exemple y avoir certains grains de café qui ne sont pas éthiopiens dans le mélange. En ce sens, le remplissement n'est pas un «tout ou rien » : il vient par degrés. Mon intention vide, la pensée de la proposition exprimée par «A est rouge» peut ainsi être partiellement remplie par l'intuition d'un A qui est vert (voir Hua XIX/2, p. 562).

L'évidence de l'intuition est un autre aspect important de la conception de Husserl. Là où le remplissement est une caractérisation fonctionnelle de ce qu'est une intuition, les différents degrés de remplissement, eux, ne correspondent pas aux différents types d'évidence en jeu dans l'intuition. Disons que je pense à une tasse en porcelaine pleine de café. Mon intention signitive n'est remplie que lorsque j'ai une représentation intuitive de cette tasse particulière. Or une tasse, comme tout objet tridimensionnel, est toujours donnée sous une certaine perspective. Le problème ici est que, même

9. L'immédiateté de l'intuition ne signifie pas que toutes les intuitions sont aussi originaires. Votre intuition demeure une intuition immédiate, même si vous ne faites que vous rappeler quelque chose, ou si vous imaginez quelque chose. La distinction entre ce qui est originaire et ce qui ne l'est pas n'affecte en aucune manière l'immédiateté de l'intuition. 
dans des conditions optimales (je perçois la tasse sous les meilleures conditions d'éclairage, à courte distance, etc.) l'intuition perceptuelle demeure quand même un type d'évidence structurellement limité. Ce type d'évidence est ouvert à révision à des stades ultérieurs de l'expérience. Dans une situation idéale, j’aurais certes une évidence parfaitement adéquate de la tasse, mais comme dans tous les cas perceptuels normaux, l'évidence est seulement plus ou moins adéquate. Mon acte de penser que les trois angles d'un triangle ont la somme de $\mathrm{I} 80$ degrés est remplie par l'édition eidétique des triangles. Mais dans ce cas, il n'y a aucun degré de remplissement. L'évidence qui caractérise un tel type de remplissement est appelée une évidence apodictique.

Bref, l'évidence est une propriété des intuitions, dont le remplissement est une fonction. C'est grâce à cette propriété que les intuitions nous fournissent une connaissance. Savoir que le couteau est sur la table, c'est avoir une intention signitive (exprimée par la proposition «le couteau est sur la table) qui est remplie adéquatement par l'intuition correspondante.

Nous l'avons vu, l'idée husserlienne que les intuitions ont pour fonction de remplir une intention de signification tire son origine de l'idée brentanienne que l'attention exercée sur des parties du contenu intuitif nous permet d'isoler un aspect ou une partie de celui-ci pour l'unifier avec des parties d'autres contenus intuitifs semblables. En observant différentes nuances de vert, je peux porter par exemple mon attention sur les pointes plus ou moins prononcées de jaune qui ressortent dans certaines nuances, et j'en viens après certaines observations à former un concept de jaune comme le concept de ce qui intervient à différents degrés d'intensité dans les nuances de vert observées. Lorsque Husserl dit que les intuitions individuelles des nuances de vert remplissent à différents degrés d'adéquation mon intention de signification exprimée dans mon jugement «je cherche du jaune dans chacune de ces nuances de vert ", Brentano suggère de manière analogue que l'attention au jaune dans les différentes observations des nuances de vert est motivée par mon intérêt d'y trouver du jaune. Les parties du contenu ainsi isolées et unifiées servent alors de médiateur (Vermittler) ou de signe qui, bien que de nature différente des intentions de signification husserliennes, jouent un rôle similaire ${ }^{10}$.

Dans sa philosophie de l'esprit, Brentano considère l'intuition comme l'élément le plus fondamental dans la théorie des représentations. Cet élément est à la base, à la fois des jugements et des actes affectifs, les deux autres classes de sa tripartition du mental. Contrairement aux jugements et aux actes d'émotion, les représentations (y compris les intuitions) n'ont pas de polarité. Je peux accepter ou rejeter l'existence de quelque chose dans le jugement, je peux aimer ou haïr quelque chose dans les actes émotifs, mais on ne retrouve rien d'analogue dans la représentation. Il y a cependant dif-

10. Ce modèle est proposé par Brentano dans ses cours de logique de Vienne des années I 880. Voir Brentano EL72, p. I2340. 
férentes sortes de représentation, distinctes les unes des autres par leur degré de clarté : la forme la plus pure de la représentation est l'intuition (Anschauung). Ces représentations ont le degré le plus élevé de clarté. Les couleurs que je vois et les sons que j'entends sont des intuitions au sens de Brentano. Toutes les autres représentations sont des représentations non intuitives. Pour suivre l'exemple de Brentano, j'ai une représentation non intuitive lorsque je pense à un carré rond. Mais j'ai aussi une représentation non intuitive lorsque je pense ou m'imagine un carré rouge.

En ce sens, on peut dire que toutes les représentations ou bien sont des intuitions ou bien sont basées sur (au sens d'être partiellement constituées par) des intuitions. Brentano soutient une thèse semblable en ce qui concerne les actes mentaux plus généralement: tous les actes mentaux sont ou bien des représentations, ou bien partiellement constitués par des représentations.

Qu'en est-il des cas où je vois d'abord un carré rouge puis que je m'imagine ensuite un carré rouge? Selon Brentano, le premier acte est une représentation purement intuitive, alors que le second est non intuitif, bien qu'il ait un certain degré d'intuitivité. Comment doit-on comprendre cela? Brentano, se basant sur Aristote, soutient que le contenu de ces actes est différent, bien qu'ils semblent avoir le même contenu ${ }^{11}$. Or c'est seulement de manière impropre (uneigentlich) — c'est-à-dire suivant ce qu'ils semblent être - qu'il est correct de dire qu'ils ont le même contenu. En fait, l'imagination a pour contenu une pensée dont le noyau est intuitif. Conséquemment, l'intuition et l'imagination ne sont pas des modes distincts de représentation, mais ils diffèrent en vertu des propriétés de leur contenu: les intuitions, par exemple, ont un degré d'intensité que les représentations non intuitives n'ont pas.

Enfin, comme nous l'avons déjà mentionné, les intuitions sont liées de manière indissoluble avec l'acceptation assertorique de ce qui est présenté dans l'intuition. L'idée est ici que je ne peux qu'accepter ce qui m'est donné intuitivement: je ne peux avoir une représentation intuitive de roses rouges et du même coup accepter ces roses rouges ou prendre position d'une certaine manière sur elles. Cette acceptation est plus primitive que l'acceptation impliquée dans le jugement, bien qu'elle lui soit similaire: dans le jugement, je peux accepter ou rejeter ce qui m'est donné, alors que je n'ai pas ce choix dans l'intuition.

\section{Trois différences principales entre Brentano et Husserl sur l'intuition}

Sur la base de l'esquisse générale de cette conception de l'intuitivité qu'on retrouve notamment chez Brentano et Husserl, nous sommes maintenant en 
position d'établir ce qu'on pourrait appeler une conception phénoménologique neutre - car commune à Brentano et Husserl — de l'intuition.

a) Elle est une forme de perception, ou elle est basée sur la perception;

b) Elle est immédiate ("présente", dans la terminologie de Husserl; "propre» dans la terminologie de Brentano);

c) Elle est une source de connaissance;

d) Elle est la base de l'imagination;

e) Elle est systématiquement accompagnée et complétée par une intention "vide» (une visée, dans la terminologie de Husserl, ou un intérêt basé sur l'attention, un "plaisir à remarquer" (Lust am Bemerken), suivant la terminologie brentanienne).

Il y a trois différences principales entre les deux conceptions présentées au $\mathbb{3}_{3}$ : la première se trouve dans le désaccord entre Husserl et Brentano sur la relation entre intuition et acceptation. Premièrement, Husserl rejette l'idée que les intuitions soient systématiquement reliées à une acceptation. Deuxièmement, il accepte deux types de perception, la deuxième étant la perception du général. Brentano et la plupart de ses élèves rejettent ce deuxième type de perception. La troisième différence, la plus importante, est que pour Husserl l'intuition est un mode de conscience, alors que pour Brentano elle est une représentation qui n'est distincte des autres représentations non intuitives que par degrés. Husserl décrit souvent l'expérience de remplissement d'une intuition comme une "conscience particulière de remplissement $(R L, V I, \mathbb{8}$, p. 694). Dans les Idées directrices en particulier - mais aussi plus tard lorsqu'il décrit l'activité de l'intuition - il parle de conscience perceptuelle. Cela doit être mis en contraste avec ce que Husserl décrivait au début de sa carrière en termes de «re-présentation" (Repräsentation), mais aussi avec ce qu'il appelle les actes signitifs. Les actes perceptuels (de remplissement) et les actes signitifs constituent, contrairement à la conception de Brentano, deux catégories d'actes fondamentalement différentes. Sur ce point, Husserl s'éloigne considérablement de Brentano. Selon ce dernier, la différence entre une intuition et une représentation non intuitive tire son origine dans la nature de l'objet présenté, et non dans le mode de conscience par lequel l'objet est donné. Selon Brentano, les intuitions sont des représentations propres, alors qu'une représentation surrogative (ma représentation d'une figure de mille côtés, par exemple) est une représentation impropre.

Cette différence est visible dans un exemple classique de Husserl. Imaginez que vous voyez une arabesque dans un livre décoré et que vous pensez d'abord voir une simple décoration ou une image, mais qu'à un certain moment vous réalisez qu'il s'agit d'un symbole linguistique. À ce moment, le mode de conscience change, dit Husserl. "Vous voyez le signe, mais vous n'y portez pas attention, vous n'en avez pas l'intuition " (Hua XXII, p. I I 5I I6). En un sens, on pourrait dire que l'objet vu s'efface un peu lorsque le 
changement s'opère: il demeure le même objet, le même contenu de perception, mais notre attitude par rapport à lui est différente.

À cela, Brentano répondrait que la seule différence est que, motivé par un intérêt changeant, vous portez alors votre attention sur une partie différente de l'objet. C'est le seul changement. L'objet ne s'efface pas comme un tout: c'est plutôt que des parties de celui-ci sont isolées afin de pouvoir porter votre attention sur d'autres parties. L'explication est simple: le signe que vous percevez est «dans» l'image, bien que vous continuiez en tout temps à voir l'image.

Si nous laissons certaines de ces différences descriptives de côté, on peut penser que la différence concernant la conception de l'intuition n'est pas particulièrement importante. Après tout, Brentano parle d'un changement d'intérêt, d'une focalisation de l'attention, là où Husserl parle d'un différent mode de conscience. Mais ce changement dans le mode de conscience est pour Husserl aussi un indicateur de l'intentionnalité. Pour Husserl, lorsque vous regardez les formes esthétiques de l'arabesque, puis lorsque vous regardez le mot qui y est écrit, le contenu ne change pas: les deux ont le même contenu intuitif. Ce qui change est l'objet perçu, et ce changement se manifeste dans les différentes manières par lesquelles le contenu intuitif est intentionné. Brentano dirait que ces «manières par lesquelles un contenu est intentionné » sont simplement des parties d'un seul tout, mais que la manière par laquelle ce tout entier est perçu ne change pas. Le mode de la relation intentionnelle demeure le même dans ce contexte.

\section{Intuition et identification}

Nous avons vu dans les deux dernières sections que la conception de l'intuition de Brentano et de Husserl tirait son origine de la perception, qu'elle était caractérisée en termes de représentation propre (Brentano) ou immédiate (Husserl), qu'elle était à la base des actes d'imagination, qu'elle était propre à remplir une intention vide (Husserl) ou à satisfaire un acte d'intérêt (Brentano), et donc qu'elle était en ce sens une source de connaissance. Si on reprend à nouveau l'intuition discutée dans la première section, où l'objet que l'on voit au loin est perçu comme étant une maison, et qui peut être exprimée par la proposition "l'objet au loin est une maison dans un pré », il semble effectivement que la conception husserlienne et brentanienne de l'intuition puisse non seulement faire intervenir plus de ressources que ne le font les défenseurs de (pos3) pour expliquer ce qui nous fait pencher pour la vérité de cette proposition, mais également établir des liens explicatifs avec une attitude plus fondamentale que l'attitude propositionnelle, qu'on pourrait appeler l'attitude objectuelle, c'est-à-dire les différents rapports d'intérêt, ou encore les rapports imaginatifs, intuitifs, médiats et immédiats qu'on entretient avec les objets. Lorsque Hildebrand parlait de l'intuition d'un état de choses en termes de Kenntnisnahme, au sens où elle implique qu'un état de choses soit donné avec ses contenus fondateurs, il présuppo- 
sait que ces contenus devaient être donnés comme plus fondamentaux que l'état de choses lui-même. Il semble que les différents rapports représentationnels dans l'attitude objectuelle, tels que nous les avons décrits en partant de Husserl et Brentano, puissent jouer ce rôle. Et s'ils le jouent, alors il semble que l'attitude des défenseurs de (pos3) par rapport à l'intuition soidisant non propositionnelle soit non seulement injustifiée, mais un obstacle important à la conception elle-même.

Quel rôle peut-on attribuer maintenant à ce concept d'intuition propre à l'attitude objectuelle, et comment celui-ci pourrait-il permettre d'éviter l'obstacle en question? Si le concept d'intuition en rapport avec l'attitude objectuelle, et tel que présenté par Brentano et Husserl, doit être lié de manière explicative avec celui d'intuition comme «semblance» de la vérité d'une proposition, on doit identifier une quelconque vertu épistémique dans ce concept d'intuition relatif à l'attitude objectuelle. Une façon de le faire est de partir du critère de réidentification discuté par Mulligan (2007, 21 5) et de l'appliquer à l'intuition. Supposons que je m'égare en forêt et perçoive à tI une maison rouge, puis que je continue mon chemin et revienne au même point, cette fois en provenance d'une autre direction, et que je revoie la même maison sous un autre angle à t2. J'ai deux intuitions distinctes d'une même maison. Qu'est-ce qui me permet d'identifier l'objet comme étant identique dans les deux intuitions? Pour un défenseur de (pos3), ma nouvelle intuition perceptuelle de la maison rouge à t 2 vient renforcer mon penchant pour la proposition «l'objet sur cette colline dans la forêt est une maison rouge ", mais dans les deux cas, tant à ti qu'à t2, le contexte perceptuel ou la similarité des objets perçus ne jouent aucun rôle dans le renforcement du penchant. Le défenseur de (pos3) est tenu, s'il croit que l'intuition est une attitude propositionnelle sui generis, d'expliquer le renforcement du penchant par le fait qu'une attitude propositionnelle à t2 porte sur la même proposition que l'attitude propositionnelle à tı. Le phénoménologue a ici plus de ressources: pour lui, si $S$ a l'intuition (épistémiquement significative) de $\mathrm{A}$, alors $S$ voit A à tı, puis à t2, comme le même objet. Donc, si j'ai la représentation intuitive, ou l'intuition (épistémiquement significative), de cette maison (ou de cette chose rouge), c'est que je vois cette chose à tI et à t2 comme étant la même chose.

Dans son adaptation du critère d'identification à l'appréhension, Mulligan propose aussi un critère qui peut cadrer avec l'intuition catégoriale de Husserl:

$S$ appréhende l'état de choses exprimé par $P$ ssi $S$ 'voit' l'état de choses exprimé par $P$ à tI et $S$ 'voit' l'état de choses exprimé par $P$ à t2, et $S$ identifie correctement $P$ à tr et $P$ à tr. ${ }^{12}$

12. Comparer avec Mulligan (2007), p. 2I 5-2I6. 
L'intuition (tant celle d'objets que d'états de choses) n'est pas ellemême une identification, mais parce qu'elle implique une identification, on peut lui attribuer au moins indirectement une vertu épistémique. C'est pour cette raison que j'ai parlé plus haut d'une intuition épistémiquement significative en contraste avec l'intuition tout court (tant celle d'objet que d'états de choses). L'intuition n'a pas elle-même de condition de correction, mais l'identification qu'elle implique a, elle, des conditions de correction. Prenons l'exemple préféré de Husserl, la statue de cire qu'on prend d'abord pour une jolie femme, puis qu'on prend ensuite pour un morceau de cire. Dans le premier cas, j'ai une intuition de $\mathrm{A}$ comme $F$. Dans le deuxième cas, j'ai une intuition de A comme $G$, mais je ne pourrais avoir cette expérience «euréka!" si je n'identifiais pas $\mathrm{A}$ à tı et $\mathrm{A}$ à t2.

Comme on l'a déjà suggéré, la situation dans les cas d'intuitions qui ne portent pas sur des objets mais sur des états de choses n'est pas foncièrement différente. Disons qu'à ti j'exprime un état de choses perçu par «la rose est rouge ", puis qu'à t2 je l'exprime différemment, par exemple par «la troisième fleur à droite dans le jardin est rouge », parce que je veux par exemple indiquer un fait à mon interlocuteur. Il s'agit dans les deux cas de deux appréhensions différentes du même état de choses exprimé par des propositions différentes. Ce cas peut être problématique pour un défenseur de (pos3) car il ne pourra pas recourir, contrairement au cas précédent, à un renforcement du "penchant» par le fait qu'une attitude propositionnelle à t2 porte sur la même proposition que l'attitude propositionnelle à tı. Or il semble ici qu'une explication du renforcement du penchant pour $P$ sur la base d'intuitions épistémiquement significatives - parce qu'impliquant une identification - soit plus fidèle à la lettre de l'idée des défenseurs de (pos3), à savoir que l'intuition que la rose est rouge s'explique centralement par le penchant ou l'inclination envers une proposition: dans le cas présent, cette inclination, renforcée à t2, est expliquée par les différentes appréhensions d'un même état de choses $^{13}$.

\section{Remarques finales}

Dans ce qui précède, nous nous sommes interrogé sur la conception de l'intuition défendue par les partisans de (pos3), selon laquelle l'intuition est un seeming, une "apparence", ou une "impression qu'une proposition est vraie. Pour les défenseurs de (pos3), l'intuition a une vertu épistémique parce que, lorsqu'il semble que $P$ à $S$ en l'absence d'une évidence contraire (defeater), $S$ est justifié de croire que $P$. Comme les défenseurs de (pos3), nous croyons qu'il y a dans l'intuition une attitude sui generis qui n'est pas réductible à la croyance, mais nous doutons que cette attitude soit strictement propositionnelle, comme ceux-ci le suggèrent, et nous doutons que la

13. L'idée que les actes de connaissance sont supportés par des synthèses d'identification est développée par Husserl (1984, 52). 
métaphore de l'inclination, du "pushed by ou du «seeming " intellectuel, si elle est censée pointer vers un phénomène important pour la théorie de la connaissance, soit vraiment à comprendre naturellement dans un sens propositionnel, en excluant d'emblée les phénomènes non propositionnels, comme le font les défenseurs de (pos3). Nous avons de plus suggéré que le rejet des formes non propositionnelles d'intuition par les défenseurs de (pos3) constituait plutôt un obstacle à l'idée de base exprimée dans (pos3). C'est pourquoi nous avons d'abord distingué différentes formes de connaissance, en partant des travaux de phénoménologues comme Husserl, Reinach, Hildebrand et Bassenge, en opérant avec eux une double distinction entre les actes de connaissance (propositionnels et non propositionnels) épisodiques, et les états de connaissance non épisodiques (propositionnels et non propositionnels). Cette double distinction nous a permis d'isoler un aspect du "seeming ", l'appréhension, que les phénoménologues analysent de manière plus détaillée que l'analyse du seeming proposée par les défenseurs de (pos3). Nous avons aussi indiqué que Brentano et Marty avaient eux aussi identifié, en ce qui concerne les objets, un phénomène apparenté à l'appréhension des phénoménologues, lui aussi dans la catégorie des connaissances épisodiques non propositionnelles.

Nous avons aussi partagé avec les défenseurs de (pos3) la conviction qu'il y a un sens substantiel à dire que les intuitions ont une vertu épistémique, et qu'une théorie des intuitions, phénoménologique ou non, devait rendre compte de ce fait. Pour ce faire, nous avons eu recours dans un premier temps à la théorie du remplissement de Husserl et à la conception de la satisfaction de l'intérêt de Brentano. Ces deux modèles font une place à la notion d'un type d'évidence qui est ouvert à des stades ultérieurs de l'expérience, de manière analogue aux "évidences contraires»(defeaters) qui servent de contrepartie à la justification dans le modèle proposé par les défenseurs de (pos3). Ensuite, nous avons eu recours aux synthèses d'identification de Husserl pour montrer que les intuitions en un sens primaire, c'est-à-dire celles qui portent sur des états de choses ou des objets, impliquent une identification qui leur octroie leur vertu épistémique. Cette conception permet du coup de rendre compte de manière unifiée du phénomène de l'intuition, tant au niveau propositionnel qu'au niveau sub-propositionnel.

De cette manière, on peut aussi rendre compte de manière unifiée du fait que l'intuition est une attitude sui generis en se basant sur des analyses qui s'appliquent non seulement aux cas propositionnels, mais en plus aux cas non propositionnels. Du coup, on peut doublement accommoder ( $\operatorname{pos}_{3}$ ) en donnant une explication de la nature sui generis des intuitions et en fournissant des outils supplémentaires à ses défenseurs pour décrire ce qu'ils appellent la phénoménologie du «seeming », tout en abandonnant son primitivisme propositionnel, qui semble être davantage un problème qu'une solution, tant pour (pos3) que pour une phénoménologie de l'attitude cognitive particulière en jeu dans l'intuition. 


\section{Bibliographie}

Bassenge, F. "Hexis und Akt. Eine phänomenologische Skizze", Philosophischer Anzeige, volume 4, numéro 2, I930, pp. I63-I 68.

Bealer, G. "Intuition and the Autonomy of Philosophy," in DePaul \& Ramsey, I998.

—. "Modal Epistemology and the Rationalist Renaissance". In Conceivability and Possibility, T. Gendler \& J. Hawthorne (eds.), New York, Oxford University Press, 2002.

Brentano, Franz (I 892). “Über ein optisches Paradoxon”. In Zeitschrift für Psychologie und Physiologie der Sinnesorgane III, pp. 349-358.

—. (I 893). "Über ein optisches Paradoxon. Zweiter Artikel”. In Zeitschrift für Psychologie und Physiologie der Sinnesorgane V, pp. 6I-82.

—. (I 894). "Zur Lehre von den optischen Täuschungen”. In Zeitschrift für Psychologie und Physiologie der Sinnesorgane VI, pp. 37-45.

-. ( ( 884 -I 885). Logikvorlesung. EL72. Manuscript. Houghton Library, Harvard University.

- (1886). Ausgewählte Fragen aus Psychologie und Ästhetik. Manuscript. Houghton Library, Harvard University.

—. (1959). Grundzüge der Ästhetik, Bern, Francke Verlag.

- ( (1987). "Von der Natur der Vorstellung" (J. Brandl, ed.). In Conceptus 2I, pp. 25-3I.

Chudnoff, E. Intuition, Oxford, Oxford University Press, 2013.

DePaul, M. \& W. Ramsey (ed.). Rethinking Intuition: The Psychology of Intuition and Its Role in Philosophical Inquiry, Lanham, MD, Rowman and Littlefield, I 998 .

Hildebrand, D. “Die Idee der sittlichen Handlung”, Jahrbuch für Philosophie und phänomenologische Forschung, volume 3, I916, pp. I26-252.

- Der Sinn des philosophischen Fragens und Erkennens, Bonn, Hanstein, I950.

Huemer, M. Skepticism and the Veil of Perception, Lanham, Rowan \& Littlefield, $200 \mathrm{I}$.

—. Ethical Intuitionism, New York, Palgrave Macmillan, 2005.

_. "Compassionate Phenomenal Conservatism", Philosophy and Phenomenological Research, volume 74, 2007, pp. 30-55.

Husserl, E. Ideen zu einer reinen Phänomenologie und phänomenologischen Philosophie (K. Schuhmann, ed.), Den Haag, Martinus Nijhoff (Hua III, I), I976. Traduction française par Paul Ricoeur, Idées directrices pour une phénoménologie, Paris, Gallimard, I985.

—. Aufsätze und Rezensionen (1890-1910) (B. Rang, ed.), Den Haag, Martinus Nijhoff (Hua XXII), I979.

- Logische Untersuchungen. Zweiter Band: Untersuchungen zur Phänomenologie und Theorie der Erkenntnis (U. Panzer, ed.), Den Haag, Martinus Nijhoff (Hua XIX, I et XIX, 2), I984.

Koksvik, O. Intuition, Dissertation, Australian National University, 20 I I.

Kraus, Oskar. “Toward a Phenomenognosy of Time Consciousness”. In Linda McAlister (ed.), The Philosophy of Franz Brentano, London, Duckworth, I976, pp. 224-239.

Lewis, D. Philosophical Papers: Volume I, New York, Oxford University Press, I983. 
Mulligan, K. "Intentionality, Knowledge and Formal Objects", Disputatio, Vol. II, $\mathrm{n}^{\circ} 23$, November 2007, pp. 205-228.

—. "Knowledge First. A German Folly? ”. In J. Dutant, D. Fassio, et A. Meylan (eds), Liber Amicorum Pascal Engel, Université de Genève, 20I4, pp. 380400.

Pust, J. “Intuition”. In Edward Zalta (ed.), Stanford Encyclopedia of Philosophy, http://plato.stanford.edu/entries/intuition/ (dernier accès 5 avril 20I7).

Reinach, A. [I9II]. "Zur Theorie des negativen Urteils”. In K. Schuhmann \& B. Smith, Sämtliche Werke (eds), Textkritische Ausgabe, 2 volumes, Vol. I, Die Werke, Munich, Philosophia Verlag, I989, pp. 95-I40.

Sosa, E. “Minimal Intuition”. In De Paul \& Ramsay, I998.

Tucker, C. (ed.). Seemings and Justification. New Essay on Dogmatism and Phenomenal Conservatism, New York, Oxford University Press, 20I3.

Williamson, T. Knowledge and its Limits, Oxford, Oxford University Press, 2000. 